\title{
The Effects of Homocysteine Level in the Critically III Patient. A Review
}

\author{
Ovidiu Horea Bedreag1,2, Alexandru Florin Rogobete ${ }^{1,2}$, Dorel Sandesc ${ }^{1,2}$, Carmen Alina Cradigati \\ Mirela Sarandan³, Radu Nartita4, Raluca Dumache², Mihai Mircea Diaconu², Marius Papurica1,2 \\ ${ }^{1}$ Clinic of Anesthesia and Intensive Care, "Pius Brinzeu" County Emergency Hospital, Timișoara, Romania \\ 2 Faculty of Medicine, "Victor Babeș" University of Medicine and Pharmacy, Timișoara, Romania \\ 3 "Casa Austria" Clinic of Anesthesia and Intensive Care, "Pius Brinzeu" County Emergency Hospital, Timișoara, Romania \\ ${ }^{4}$ Faculty of Chemistry, Biology and Geography, West University of Timișoara, Romania
}

\section{CORRESPONDENCE}

Alexandru Florin Rogobete
Clinic of Anesthesia and Intensive
Care, "Pius Brinzeu" County
Emergency Hospital
156 Liviu Rebreanu Bd
300736 Timișoara, Romania
Tel: +40 356433111
E-mail: alexandru.rogobete@umft.ro

\section{ARTICLE HISTORY}

Received: 5 June, 2016

Accepted: 15 June, 2016
Ovidiu Horea Bedreag • 156 Liviu Rebreanu Bd, 300736 Timișoara, Romania, Tel: +40 356433111 Dorel Sandesc • 156 Liviu Rebreanu Bd, 300736 Timișoara, Romania, Tel: +40 356433111

Carmen Alina Cradigati • 156 Liviu Rebreanu Bd, 300736 Timișoara, Romania, Tel: +40 356433111 Mirela Sarandan • 156 Liviu Rebreanu Bd, 300736 Timișoara, Romania, Tel: +40 356433111

Radu Nartita 4 Vasile Pârvan Bd, 300223 Timișoara, Romania, Tel: +40 256592111

Raluca Dumache • 156 Liviu Rebreanu Bd, 300736 Timișoara, Romania, Tel: +40 356433111

Mihai Mircea Diaconu • 156 Liviu Rebreanu Bd, 300736 Timișoara, Romania, Tel: +40 356433111

Marius Papurica • 156 Liviu Rebreanu Bd, 300736 Timișoara, Romania, Tel: +40 356433111

\begin{abstract}
Increased levels of homocysteine (HCYS) represent a risk factor for a series of physiopathological conditions: mental retardation, cardiovascular and neurodegenerative diseases, Parkinson's and Alzheimer's disease, depression, osteoporosis, endothelial dysfunction and inhibition of cell proliferation. This paper aims to present the pathophysiological implications of HCYS and the correlation of hyperhomocysteinemia (H-HCYS) with critical condition in the intensive care unit (ICU). Hypovitaminosis B and folate deficiency is directly involved in the inhibition of HCYS metabolism and the accumulation of HCYS in the plasma and tissues. Critically ill patients are more prone to H-HCYS due to hypermetabolism and accelerated synthesis produced by reactive oxygen species (ROS). In conclusion it can be affirmed that the determination and monitoring of HCYS plasma levels may be of interest in optimizing the therapy for critically ill patients. Moreover, by controlling HCYS levels, and implicitly the essential cofactors that intervene in the specific biochemical pathways, such as vitamin B6, vitamin B12 and folic acid can provide a diversified and personalized treatment for each patient.
\end{abstract}

Keywords: homocysteine, hypovitaminosis, oxidative stress, intensive care unit

\section{INTRODUCTION}

Homocysteine (HCYS) is a non-essential amino acid resulted from the breakdown of methionine. ${ }^{1}$ In the remethylation reaction of HCYS are involved a series of cofactors, such as pteroylglutamic acid (folic acid), cobalamin (vitamin B12) and pyridoxine (vitamin B6). ${ }^{2}$ HCYS normal plasma concentration is 5-14 $\mu \mathrm{mol} / \mathrm{L} .^{3}$

Therefore, low cofactor concentration leads to the inhibition of the metabolic cycle. HCYS accumulation in the body (hyperhomocysteinemia, H-HCYS) entails multi-organ pathologies: neurological diseases - mental retardation, ${ }^{4}$ cerebral atrophy, ${ }^{5}$ seizures,${ }^{6}$ depression, ${ }^{7}$ Alzheimer's disease, ${ }^{8}$ Parkinson's disease, ${ }^{9}$ ophthalmic abnormalities, ${ }^{10}$ bone disease, ${ }^{11}$ and cardiovascular disease. ${ }^{12}$ This paper presents an update on the biochemical mechanisms and the pathophysi- 


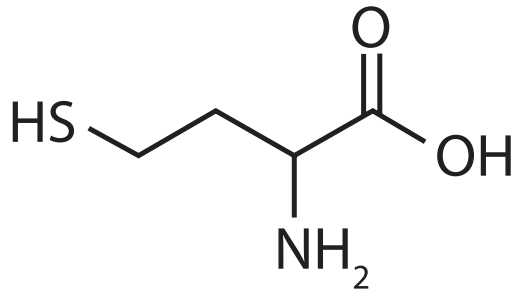

FIGURE 1. The chemical structure of HCYS

ological implications of H-HCYS in patients with certain diseases, including polytrauma patients.

\section{BIOCHEMICAL PROPERTIES OF HCYS}

\section{Structural aspects}

From a chemical point of view, HCYS is a non-proteinogenic amino acid. ${ }^{12}$ It is structurally different from cysteine by a methylene group. According to the International Union of Pure and Applied Chemistry (IUPAC), HCYS is defined as 2-amino-4-sulfanylbutanoic acid, ${ }^{3,12}$ with the molecular formula $\mathrm{C}_{4} \mathrm{H}_{9} \mathrm{NO}_{2} \mathrm{~S}$ (Figure 1).

\section{HCYS metabolism}

HCYS is biosynthesized only in cells, from methionine. ${ }^{13}$ Methionine is an essential amino acid, while cysteine is biosynthesized by transfering a sulfur atom from methionine to the hydroxyl group of serine. Through the action of methionine-adenosyl-transferase on methionine, S-adenosyl methionine is produced, which through the intervention of methyl-transferase produces S-adenosyl-HCYS and a free methyl group $\left(-\mathrm{CH}_{3}\right) .6$ Finally, adenosyl-homocystienase converts S-adenosyl-HCYS to HCYS, releasing a molecule of adenosine. ${ }^{14,15}$

HCYS excess is captured by the kidneys and liver, where it is metabolized. ${ }^{16}$ HCYS is filtered at the glomerulus and absorbed by tubular uptake. The following enzymes: cystathione- $\beta$-synthase, cystathione- $\gamma$-lyase, cysteine aminotransferase, 3-mercaptopyruvate-sulfur-transferase are contained in the kidneys and convert HCYS to methionine through remethylation. ${ }^{14-17}$ The liver also contains the enzymes necessary for HCYS catabolism. In this regard, liver and kidney disorders lead to H-HCYS. Another important factor in the accumulation of homocysteine in the plasma is protein-energy malnutrition. ${ }^{16,17}$

The biochemical reactions that regulate the levels of HCYS are remethylation and transsulfuration. ${ }^{17}$ On the remethylation way, a methyl group is transferred via methylcobalamin/betaine. The methyl group is generated by the active form of folic acid (5-methyltetrahydrofolate) or by S-adenosylmethionine. After remethylation, methionine can be reused for the production of S-adenosylmethionine, which plays an important role in several biochemical reactions: DNA methylation, synthesis of carnitine, coenzyme A and melatonin synthesis. ${ }^{18} \mathrm{HCYS}$ remethy-lation uses two enzymes: methionine-synthase and betaine-homocysteine-S-methyl-transferase. The action of these enzymes is regulated by two factors: vitamin B12, cofactor for methionine-synthase, and betaine, cofactor for betaine-homocysteine-S-methyl-transferase. Methionine thus formed is successively activated by the action of adenosine triphosphate (ATP) and adenosyltransferase, forming S-adenosylmethionine. The latter, through a transmethy-lation reaction, generates a methyl group, and through the action of S-adenosylmethionine-decarboxylase it is decarboxylated contributing to polyamine synthesis. On the transsulfuration pathway HCYS is converted to cysteine and taurine by the action of the following enzymes: cystathione- $\beta$-synthase and cystathione- $\gamma$-lyase. These enzymes have as cofactor the active form of vitamin B6 (pyridoxal-5-phosphate). The decarboxylation reaction of cysteine produces cysteamine. Cysteamine is a precursor in the biosynthesis of coenzyme A or taurine (Figure 2).

Also, in the case of urgently required energy, the metabolism of HCYS can be transferred to the formation of a-keto-butyrate, and finally obtaining succinyl-CoA.

\section{PATHOPHYSIOLOGICAL EFFECTS OF HYPERHOMOCYSTEINEMIA (H-HCYS)}

\section{H-HCYS and neurological disorders}

Studies have shown that a high level of HCYS is associated with a high incidence of atherosclerosis. Cerebral small vessel disease ${ }^{19}$ is associated with a number of diseases: hypertension, macro- and microvascular disease, endothelial dysfunction ${ }^{20}$ and leakage of the blood-brain barrier. ${ }^{5}$ Kloppenborg et al. reported that patients with two or more atherosclerotic lesions have abnormal HCYS. Also, increased HCYS concentration is directly proportional to the degree of white matter lesions ${ }^{5}$ and lacunar infarcts. ${ }^{21}$

Other neurological disorders that present an increased HCYS plasmatic concentration are Alzheimer's disease, ${ }^{8,22,23}$ Parkinson's disease, ${ }^{9,24}$ dementia, cognitive decline, ${ }^{6}$ schizophrenia, ${ }^{25}$ depression and migraine. ${ }^{14,26} \mathrm{An}$ other condition related to high levels of HCYS reported by many studies is ophthalmic vascular disease. Allam et 


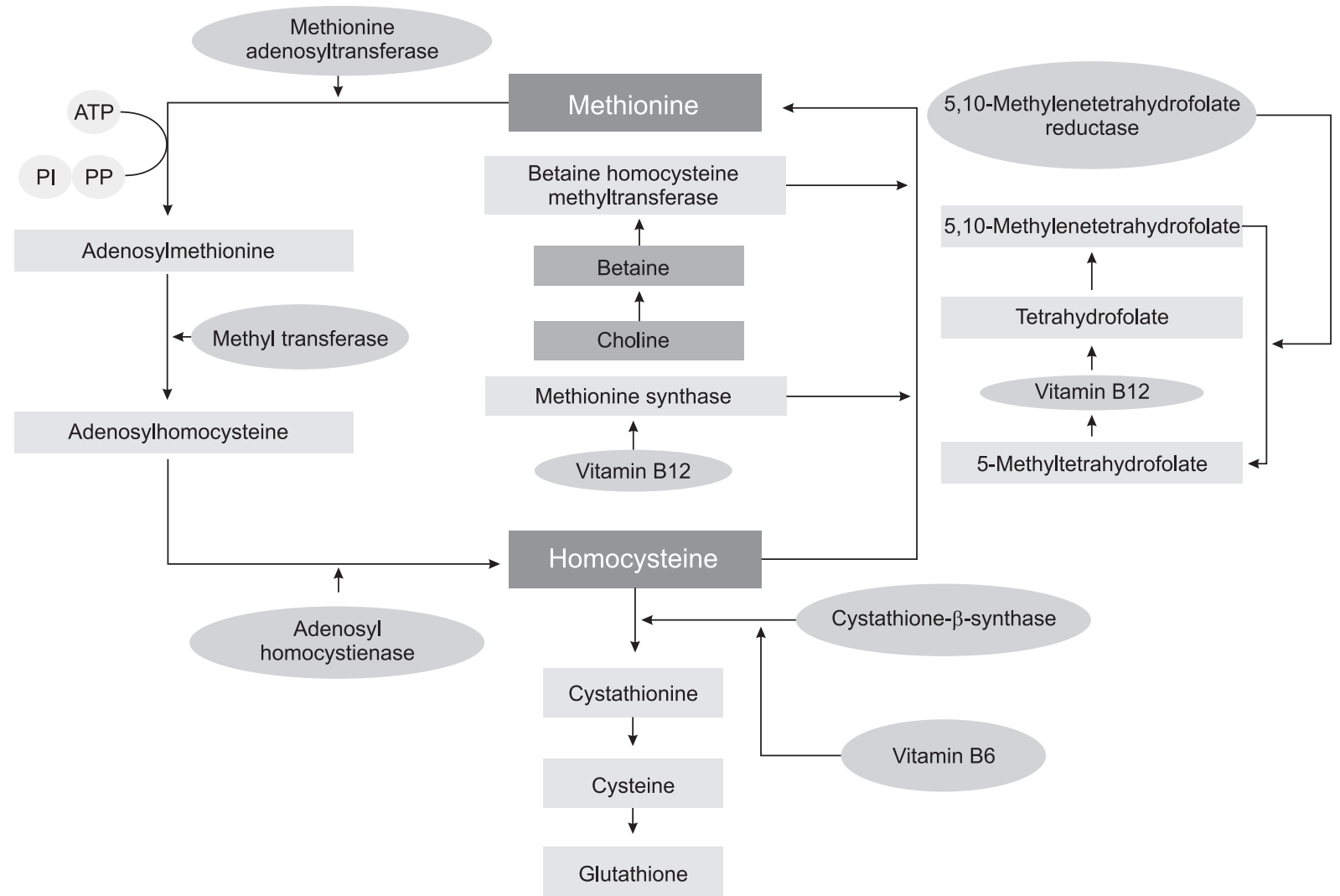

FIGURE 2. The biochemical pathway of homocysteine. Methionine is converted into S-adenosylmethionine through the action of methionine adenosyltransferase. Subsequently, it is being demethylated to S-adenosylhomocysteine. Through the action of adenosylhomocysteine homocystienase is produced. Homocysteine is converted by the action of cystathionine- $\beta$-synthase enzyme and of vitamin B6 into glutathione. Another way of remethylation of homocysteine into methionine is represented by the folate pathway through the action of methionine synthase and vitamin B12. Also, in the liver and kidneys, homocysteine is remethylated in methionine through betainehomocysteine methyltransferase.

al. conducted a study on egyptian patients diagnosed with Behcet's disease which had high plasma levels of HCYS, that led to the conclusion that, in these patients, the accumulation of HCYS is directly implicated in eye injuries, such as retinal atrophy, vitreousum hemorrhage, retinal detachment and vasculopathic complications. ${ }^{10}$

The main neurotoxic effects are caused by cytosolic calcium accumulation, which activates several neurodegenerative kinases, as a consequence to hydrogen preoxid production which can degrade DNA and potentiate neuronal beta-amyloid protein synthesis. ${ }^{19}$ Vitamin B and folate deficiency, which are directly involved in the metabolism of methionine as cofactors, also have neurodegenerative effects. ${ }^{19,27,28}$ Folate deficiency in neuroblastoma cells, ${ }^{14}$ inducing free oxygen radicals simultaneously with H-HCYS, lead to memory loss up to $66 \% .{ }^{29}$ Patients with Alzheimer's and Parkinson's disease have high levels of HCYS and low levels of vitamin B and folate. ${ }^{22,23}$ Patients diagnosed with schizophrenia have also been reported to have very high levels of HCYS together with folate deficiency and normal levels of vitamin B. ${ }^{25,30}$

Neuronal death and neuronal DNA structural changes are caused by DNA hypomethylation, ${ }^{31}$ due to the significant decrease of two enzymes: methyl-transferase and Sadenosyl-homocysteinase. ${ }^{32} \mathrm{H}$-HCYS poses a risk for cerebral venous thrombosis, as it is an independent factor for atherothrombotic disease. ${ }^{5,33,34}$ Numerous studies demonstrated that there is a direct proportionality between HCYS accumulation in the plasma, vitamin B deficiency and cerebral venous thrombosis. ${ }^{35}$ Studies by Nagaraja $e t$ al. on indian patients showed that the correlation between cerebral venous thrombosis and H-HCYS, as well as folate deficiency is statistically significant $(\mathrm{OR}=10.8) .{ }^{26}$

\section{H-HCYS and cardiovascular diseases}

HCYS accumulation in the plasma can be correlated with cardiovascular disease ${ }^{36,37}$ caused by endothelial dysfunc- 
tion, low density lipoprotein oxidation and monocyte adhesion. ${ }^{33}$ All these physiological and biochemical dysfunctions cause peripheral artery disease, ${ }^{38}$ myocardial infarction and coronary atherosclerotic disease. ${ }^{20} \mathrm{H}$-HCYS is associated with peripheral arthery disease especially in men, asopposed to women, due to hormonal differences. ${ }^{33,39}$ In menopausal women, however, HCYS rises above the normal range and can reach or exceed the serum levels of male subjects.

Coronary artery disease has a high mortality all over the world, its incidence being affected by numerous endo-genous and exogenous factors like: stress, nutrition, hormonal and metabolic dysfunction, xenobiotics, and not least genetic determinism. Some studies describe the relationship between hypovitaminosis B and folic acid deficiency related to H-HCYS and cardiovascular disease. ${ }^{3,40}$

Schaffer et al. demonstrated in a prospective cohort study on 3,056 patients that the level of HCYS is proportional to the incidence of coronary artery disease. ${ }^{3}$ They confirmed the strong relationship between H-HCYS and coronary artery disease, the correlation between the two variables being statistically significant. ${ }^{3} \mathrm{H}$-HCYS is frequently associated with certain pathologies of the cardiovascular system such as endothelial dysfunction, ${ }^{3}$ reduction of nitric oxide (NO) bioactivity ${ }^{34}$ and pro-atherogenic mechanism within the blood vessel. Stoiser et al. show that endothelial cell damage has been associated with the presence of laminin (high molecular weight glycoprotein). Through the action of HCYS on the fibronectin-fibrillin-1 complex structural and functional modifications of the cell are being produced..$^{41}$ The population of interest was divided into three subgroups according to HCYS tertiles, subgroup 1 with a value lower than $13.3 \mathrm{nmol} / \mathrm{mL}$, subgroup 2 with a value between $13.3 \mathrm{nmol} / \mathrm{mL}$ and $18.2 \mathrm{nmol} / \mathrm{mL}$, and subgroup 3 with a value higher than $18.2 \mathrm{nmol} / \mathrm{mL} .^{3}$ Another cardiovascular disorder reported in the literature as being caused by H-HCYS is spontaneous cervical artery dissection with an incidence of 2.6 from 100,000 cases. ${ }^{42}$

\section{$\mathrm{H}$-HCYS and bone disease}

Numerous studies in the literature point out the risk of osteoporosis and bone fractures as a result of increased HCYS associated with oxidative stress, the production of free radicals and the inhibition of metabolic regeneration. ${ }^{43-45}$ It has been shown that H-HCYS inhibits the biosynthesis of bone tissue. This process relies on osteoclasts destroying old bone and osteoblasts participating in the biosynthesis of new tissue. ${ }^{43}$ The biomechanics are regulated by a number of factors - hormones, cytokines, inter- leukins, and are inhibited by free radicals, collagen linked to HCYS $^{29}$ and the decrease of bone vasculature. ${ }^{46}$

Tyagi et al. reported in an experimental model a significant link between bone loss and H-HCYS. ${ }^{46}$ Kuyumcu et al. conducted a cross-sectional study including a group of 2,190 patients ${ }^{43}$ regarding the implications of endogenous antioxidants and the implications of H-HCYS regarding bone density. Uric acid is blocking the action of free radicals through his antioxidant capacity. In the studied patients low seric level of uric acid were correlated with a low action of xanthine oxidase. Bilirubine is the main blocker of the lipoprotein oxidation redox cycle, also having a high immunomodulatory capacity. Albumin has an antioxidant role, mainly given by its capacity of forming a complex with free radicals, thus inactivating their action. These three endogenous antioxidants are associated with a low incidence of osteoporosis, most likely due to their capacity of equilibrating the redox status. In the opposite sense, high levels of HCYS are correlated with a decrease of bone mineral density, and implicitly with an elevated level of osteoporosis. ${ }^{43}$

Herramann et al. confirmed that osteoporosis and metabolic bone quality are negatively affected by the accumulation of HCYS. ${ }^{29}$ Also, Enneman et al. confirm that arthritis is directly related to H-HCYS. ${ }^{31}$

\section{The relationship between H-HCYS and critically ill patients}

Patients with prolonged stay in the ICU frequently develop sepsis ${ }^{47}$ which may progress to septic shock and multiple organ failure (MODS). Ploder et al. report high levels of HCYS in patients with multiple trauma or sepsis. Studies show that H-HCYS and thrombophilia are key factors in developing septic shock. ${ }^{48}$ HCYS influences clotting factors, partialy inhibiting the coagulation cascade, particularly Factor V, and stimulates the excessive production of oxygen free radicals. ${ }^{49-51}$ Patients hospitalized in the ICU ${ }^{52}$ are more prone to the negative effect of H-HCYS due to organ dysfunction and nutritional deficiencies. . $^{9,31}$

HCYS level becomes markedly increased after 4 days of hospitalization in the ICU (compared to day 0). ${ }^{47}$ This can be explained by the altered metabolic status and systemic inflammatory response syndrome (SIRS), sepsis and multiple organ dysfunction syndrome. Renal impairment also contributes to increasing levels of HCYS. ${ }^{53} \mathrm{~Pa}$ tients with multiple trauma often develop post-traumatic depression, which itself leads to increased plasma levels of HCYS, ${ }^{54}$ being frequently associated with reduced physiological and metabolic capacity. B vitamins and fo- 
late are key substrates in different metabolic cycles - the synthesis of methionine, depression-related serotonin, dopamine and norepinephrine. ${ }^{55}$ The literature demonstrates that B hypovitaminosis and low folate induce clinical depression in most patients. Pascoe et al. demonstrated that patients with prolonged stay in the ICU develop malnutrition due to hypercatabolism, ${ }^{56}$ resulting in the inhibition of several metabolic cycles that lead to clinical depression. ${ }^{57,58}$ All these disorders, combined with high levels of HCYS and serious deficiencies of B vitamins and folate lead to decreased survival in patients with multiple trauma.

\section{H-HCYS control in critically ill patients}

At present, various pharmaceutical preparations that can reduce HCYS are being studied. Supplementation with $\mathrm{B}$ vitamins and folate is an important alternative, ${ }^{59}$ but it proved not to be sufficiently effective in reducing dysfunctions induced by H-HCYS. Recently a remarkable compound has been introduced that inhibits HCYS anethole dithiolethione. ${ }^{36}$ Another compound with promising effect is resveratrol (3,5,4 '-trihidroxystilbene). ${ }^{60} \mathrm{Koz}$ et $a l$. highlighted a number of beneficial effects: it prevents apoptosis, has antioxidant properties and decreases inflammation by inhibiting nuclear factor-kappa B (NF$\mathrm{kB}$ ). The administration of resveratrol to laboratory mice decreased the level of HCYS and progressively improved tissue sections of the aorta. In addition, resveratrol is involved in complex biochemical mechanisms, stabilizing DNA replication and recombination, thereby preventing the neurodegenerative actions of H-HCYS. ${ }^{60}$ Ohashi et al. demonstrated the inhibitory action of ginsenoside Rb1 upon HCYS, a ginseng compound. ${ }^{61}$ This compund is preventing the oxidative consequences, tissue damage and neurodegenerative effects.

\section{CONCLUSIONS}

HCYS induces degenerative changes in many organs and systems. HCYS accumulation produces a series of dysfunctions that lead to impaired health status and quality of life. Degenerative cardiovascular diseases, the first cause of mortality in the world, are potentiated by H-HCYS. The main mechanisms are: increased concentration of the reactive oxygenated species, DNA damage, abnormal cell replication and impaired vascular endothelium. Cognitive diseases - Parkinson's and Alzheimer's disease, ischemic stroke and depression are also potentiated by H-HCYS. The survival rate of patients with multiple trauma is de- creased further, due to increased levels of HCYS caused by malnutrition, SIRS, sepsis, MODS, which occur frequently in ICU patients.

Current research on H-HCYS recommends resveratrol, ginsenoside $\mathrm{Rb} 1$ and anethole dithiolethione as promising substances having anti $\mathrm{H}$-HCYS properties besides supplementing with vitamin $\mathrm{B}$ and folate. Research is ongoing and of great interest given the necessary social costs to treat degenerative diseases. In conclusion, H-HCYS studies are of great interest regarding the mechanisms of action, fighting therapies, as well as the introduction of a screening to determine HCYS in subjects with cognitive impairment and degenerative cardiovascular, bone and eye disorders, or those with genetic determinism for them.

\section{CONFLICT OF INTEREST}

The authors declare that they have no competing interest.

\section{REFERENCES}

1. Miller AL, Kelly GS. Homocysteine Metabolism: Nutritional Modulation and Impact on Health and Disease Homocysteine. Alt Med Rev. 1997;2(4):234254.

2. Eldeen ON, Abd Eldayem SM, Shatla RH, Omara N, Elgammal SS. Homocysteine, folic acid and vitamin B12 levels in serum of epileptic children. Egypt J Med Hum Genet. 2012;13(3):275-280.

3. Schaffer A, Verdoia M, Cassetti E, Marino P, Suryapranata H, De Luca G. Relationship between homocysteine and coronary artery disease. Results from a large prospective cohort study. Thromb Res. 2014.134:288-93.

4. Christie L, Riedel G, Algaidi S, Whalley LJ, Platt B. Enhanced hippocampal long-term potentiation in rats after chronic exposure to homocysteine. Neurosci Lett. 2005;373:119-124.

5. Kloppenborg RP, Nederkoorn PJ, van der Graaf Y, Geerlings MI. Homocysteine and cerebral small vessel disease in patients with symptomatic atherosclerotic disease. The SMART-MR study. Atherosclerosis. 2011;216(2):461-466.

6. de Jager $\mathrm{C}$. Critical levels of brain atrophy associated with homocysteine and cognitive decline. Neurobiol Aging. 2014;35:S35-S39.

7. Jendricko T, Vidović A, Grubisić-llić M, Romić Z, Kovacić Z, Kozarić-Kovacić D. Homocysteine and serum lipids concentration in male war veterans with posttraumatic stress disorder. Prog Neuropsychopharmacol Biol Psychiatry. 2009;33(1):134-140.

8. Douaud G, Refsum H, Jager CA De, Jacoby R, Nichols TE. Preventing Alzheimer's disease-related gray matter atrophy by B-vitamin treatment. Gwenaëlle Douaud. 2013;110:9523-9528.

9. Song IU, Kim JS, Park IS, et al. Clinical significance of homocysteine (hcy) on dementia in Parkinson's disease (PD). Arch Gerontol Geriatr. 2013;57(3):288-291.

10. Allam A, Ammar H, Radwan A. Serum homocysteine level and eye involvement in Egyptian patients with Behçet's disease. Egypt Rheumatol. 2014;36(1):29-34.

11. Wijngaarden JP Van, Doets EL, Szczeci N, et al. Vitamin B 12, Folate, Homocysteine, and Bone Health in Adults and Elderly People: A Systematic Review with Meta-Analyses. J Nutr Metab. 2013;2013.

12. Ramakrishnan S, Sulochana KN, Lakshmi S, Selvi R, Angayarkanni N. Biochemistry of homocysteine in health and diseases. Indian J Biochem Biophys. 2006;43:275-283.

13. Ruseva S, Lozanov V, Markova P, Girchev R, Mitev V. In vivo investigation of homocysteine metabolism to polyamines by high-resolution accurate mass spectrometry and stable isotope labeling. Anal Biochem. 2014;457:38-47

14. Lippi G, Mattiuzzi C, Meschi T, Cervellin G, Borghi L. Homocysteine and migraine. A narrative review. Clin Chim Acta. 2014;433:5-11. 
15. Tehlivets O, Malanovic N, Visram M, Pavkov-Keller T, Keller W. S-adenosylL-homocysteine hydrolase and methylation disorders: Yeast as a model system. Biochim Biophys Acta - Mol Basis Dis. 2013;1832(1):204-215.

16. Maclean KN, Sikora J, Kožich V, et al. Cystathionine beta-synthase null homocystinuric mice fail to exhibit altered hemostasis or lowering of plasma homocysteine in response to betaine treatment. Mol Genet Metab. 2010;101:163-171.

17. Meng X, Ye W, Wang S, et al. A ratiometric two-photon fluorescent probe for cysteine and homocysteine in living cells. Sensors Actuators, B Chem. 2014;201:520-525.

18. Wei X, Gong C, Gou M, et al. Biodegradable poly(epsilon-caprolactone)poly(ethylene glycol) copolymers as drug delivery system. Int J Pharm. 2009;381(1):1-18

19. Choe YM, Sohn BK, Choi HJ, et al. Association of homocysteine with hippocampal volume independent of cerebral amyloid and vascular burden. Neurobio/ Aging. 2014;35(7):1519-1525.

20. Lan T-H, Xu Z-W, Wang Z, Wu Y-L, Wu W-K, Tan H-M. Ginsenoside Rb1 prevents homocysteine-induced endothelial dysfunction via PI3K/AKt activation and PKC inhibition. Biochem Pharmacol. 2011;82(2):148-155.

21. Jeong S-K, Kim D-H, Cho Yl. Homocysteine and pulsatility index in lacunar infarction. Clin Neurol Neurosurg. 2011;113(6):459-463.

22. Agnati LF, Genedani S, Rasio G, et al. Studies on homocysteine plasma levels in Alzheimer's patients. Relevance for neurodegeneration. J Neural Transm. 2005;112:163-169.

23. Genedania S, Rasiob G, Cortellic P, et al. Studies on Homocysteine and Dehydroepiandrosterone Sulphate Plasma Levels in Alzheimer's Disease Patients and in Parkinson's Disease Patients. Neurotox Res. 2004;6(4):327-332.

24. Jami M-S, Pal R, Hoedt E, Neubert T, Larsen JP, Møller SG. Proteome analysis reveals roles of L-DOPA in response to oxidative stress in neurons. BMC Neurosci. 2014;15:93.

25. Bouaziz N, Ayedi I, Sidhom O, et al. Plasma homocysteine in schizophrenia: Determinants and clinical correlations in Tunisian patients free from antipsychotics. Psychiatry Res. 2010;179:24-29.

26. Nagaraja D, Noone ML, Bharatkumar VP, Christopher R. Homocysteine, folate and vitamin $\mathrm{B}(12)$ in puerperal cerebral venous thrombosis. J Neurol Sci. 2008;272(1-2):43-47.

27. Glover AT, Shaw SM, Williams SG, Fildes JE. Can inflammation be an independent predictor of depression? Brain Behav Immun. 2010;24(2):173;174-175.

28. Steptoe A, Hamer M, Chida Y. The effects of acute psychological stress on circulating inflammatory factors in humans: a review and meta-analysis. Brain Behav Immun. 2007;21(7):901-912.

29. Obeid R, Herrmann W. Mechanisms of homocysteine neurotoxicity in neurodegenerative diseases with special reference to dementia. FEBS Lett. 2006;580:2994-3005

30. Möller M, Du Preez JL, Viljoen FP, Berk M, Emsley R, Harvey BH. Social isolation rearing induces mitochondrial, immunological, neurochemical and behavioural deficits in rats, and is reversed by clozapine or $\mathrm{N}$-acetyl cysteine. Brain Behav Immun. 2013;30:156-167.

31. Enneman a W, Swart KM a, Zillikens MC, et al. The association between plasma homocysteine levels and bone quality and bone mineral density parameters in older persons. Bone. 2014;63:141-146.

32. Ataie A, Ataee R, Mansoury Z, Aghajanpour M, Sciences M. Homocysteine Intracerebroventricular Injection Induces Apoptosis in the Substantia Nigra Cells and Parkinson's Disease Like Behavior in Rats. Int J Mo/ Cell Med. 2013;(3):2-7.

33. Bertoia ML, Pai JK, Cooke JP, et al. Plasma homocysteine, dietary B vitamins, betaine, and choline and risk of peripheral artery disease. Atherosclerosis. 2014;235(1):94-101.

34. Schnabel R, Lackner KJ, Rupprecht HJ, et al. Glutathione peroxidase-1 and homocysteine for cardiovascular risk prediction: results from the AtheroGene study. J Am Coll Cardiol. 2005;45(10):1631-1637.

35. Maric T, Woodside B, Luheshi GN. The effects of dietary saturated fat on basal hypothalamic neuroinflammation in rats. Brain Behav Immun. 2014;36:35-45

36. Giustarini D, Fanti P, Sparatore A, Matteucci E, Rossi R. Anethole dithiolethione lowers the homocysteine and raises the glutathone levels in solid tissues and plasma of rats: A novel non-vitamin homocysteinelowering agent. Biochem Pharmacol. 2014;89(2):246-254.

37. Amorim De Farias Leal A, Camêlo Palmeira Á, et al. Homocysteine: cardiovascular risk factor in children and adolescents? Rev Assoc Med Bras. 2013;59(6):622-628.
38. Santos MERC, Das CLE Silva F, Gomes KB, et al. Mutations in methylenetetrahydrofolate reductase and in cysthationine beta synthase: Is there a link to homocysteine levels in peripheral arterial disease? Mol Biol Rep. 2011:38:3361-3366.

39. Zoccolella S, Tortorella C, laffaldano P, et al. Elevated plasma homocysteine levels in patients with multiple sclerosis are associated with male gender. J Neurol. 2012;259:2105-2110.

40. Liu Y, Tian T, Zhang H, Gao L, Zhou X. The effect of homocysteinelowering therapy with folic acid on flow-mediated vasodilation in patients with coronary artery disease: A meta-analysis of randomized controlled trials. Atherosclerosis. 2014;235(1):31-35

41. Stoiser B, Thalhammer F, El-Menyawi I, et al. Homocysteine and laminin are not prognostic markers in patients with septic inflammatory response syndrome. Clin Diagn Lab Immunol. 2000;7(1):119-121.

42. Konrad C, Müller G a., Langer C, et al. Plasma homocysteine, MTHFR C677T, CBS 844ins68bp, and MTHFD1 G1958A polymorphisms in spontaneous cervical artery dissections. J Neurol. 2004;251(10):1242-1248.

43. Kuyumcu ME, Yesil Y, Oztürk ZA, et al. The association between homocysteine (hcy) and serum natural antioxidants in elderly bone mineral densitometry (BMD). Arch Gerontol Geriatr. 2012;55(3):739-743.

44. Poloni S, Leistner-Segal S, Bandeira IC, et al. Body composition in patients with classical homocystinuria: body mass relates to homocysteine and choline metabolism. Gene. 2014;546(2):443-447.

45. Rehackova P, Skalova S, Kutilek S. Serum homocysteine levels in children and adolescents with impaired bone health. Rev Bras Reumatol. 2013;53(6):464-469.

46. Tyagi N, Vacek TP, Fleming JT, Vacek JC, Tyagi SC. Hyperhomocysteinemia decreases bone blood flow. Vasc Health Risk Manag. 2011;7:31-35.

47. Ploder M, Kurz K, Spittler A, Neurauter G, Roth E, Fuchs D. Early increase of plasma homocysteine in sepsis patients with poor outcome. Mol Med. 2010;16:498-504.

48. Omar A, El-Shahat N, Ramadan MM. Cardiac functions in patients with sepsis and septic shock. Egypt Hear J. 2012;64(4):191-196.

49. Bramlett K, Onel E, Viscusi ER, Jones K. A randomized, double-blind, doseranging study comparing wound infiltration of DepoFoam bupivacaine, an extended-release liposomal bupivacaine, to bupivacaine $\mathrm{HCl}$ for postsurgical analgesia in total knee arthroplasty. Knee. 2012;19(5):530536.

50. Lee LM, Chang LC, Wrobleski S, Wakefield TW, Yang VC. Low molecular weight protamine as nontoxic heparin/low molecular weight heparin antidote (III): preliminary in vivo evaluation of efficacy and toxicity using a canine model. AAPS PharmSci. 2001;3(2):E19.

51. Wang C, Chen Z, Zhang T, et al. Elevated plasma homocysteine level is associated with ischemic stroke in Chinese hypertensive patients. Eur J Intern Med. 2014;25(6):538-544.

52. Zeckey C, Dannecker S, Hildebrand F, et al. Alcohol and multiple trauma: is there an influence on the outcome? Alcohol. 2011;45(3):245-251.

53. Shuwei D, Shuwen L, Xuefeng $S$, et al. Potential association of hyperhomocysteinemia with the. Chim Med J. 2014;127(10).

54. Shepherd L, Wild J. Emotion regulation, physiological arousal and PTSD symptoms in trauma-exposed individuals. J Behav Ther Exp Psychiatry. 2014;45(3):360-367.

55. Ontario HQ. Vitamin B12 and cognitive function: an evidence-based analysis. Ont Health Technol Assess Ser. 2013;13(23):1-45.

56. Pascoe MC, Crewther SG, Carey LM, Noonan K, Crewther DP, Linden T. Homocysteine as a potential biochemical marker for depression in elderly stroke survivors. Food Nutr Res. 2012;1:1-5.

57. Fazal N, Shelip A, Alzahrani AJ. Burn-injury affects gut-associated lymphoid tissues derived CD4+ T cells. Results Immunol. 2013;3:85-94.

58. Moorthy D, Peter I, Scott TM, et al. Status of Vitamins B-12 and B-6 but Not of Folate, Homocysteine, and the Methylenetetrahydrofolate Reductase C677T Polymorphism Are Associated with Impaired Cognition and Depression in Adults. J Nutr. 2012;142(8):1554-1560.

59. Nachum-biala Y, Troen AM. B-vitamins for neuroprotection: Narrowing the evidence gap. Biofactors. 2012;38(2):145-150.

60. Koz ST, Etem EO, Baydas G, et al. Effects of resveratrol on blood homocysteine level, on homocysteine induced oxidative stress, apoptosis and cognitive dysfunctions in rats. Brain Res. 2012;1484:29-38.

61. Ohashi R, Yan S, Mu H, et al. Effects of homocysteine and ginsenoside Rb1 on endothelial proliferation and superoxide anion production. J Surg Res. 2006;133(2):89-94. 